\title{
AVALIAÇÃO DA CAPACIDADE FUNCIONAL DE PACIENTES ATENDIDOS EM UM AMBULATÓRIO PERIOPERATÓRIO DO DISTRITO FEDERAL
}

\author{
Evaluation of the functional capacity of patients attended \\ in a perioperative outpatient clinic of the Federal District
Evaluación de la capacidad funcional de pacientes atendidos en un ambulatorio perioperatorio del Distrito Federal

Jacqueline Ramos de Andrade Antunes Gomes ${ }^{1 *} \oplus$, Thaís Lôbo Campos ${ }^{2} \oplus$, Glayson Carlos Miranda Verner ${ }^{3} \oplus$, Leonília Brelaz de Abreu ${ }^{4}$, Ana Caroline de Mendonça Motta ${ }^{4} \odot$, Weslei da Silva Araújo ${ }^{5} \odot$, Letícia Santos Ribeiro ${ }^{6}$, Rafael Villela Silva Derré Torres ${ }^{7} \oplus$, Anna Karoline Carvalho Sousa ${ }^{6} \oplus$, Tâmela Beatniz Matinada da Silva ${ }^{6} \oplus$, Janaina Ferreira Passos ${ }^{6} \oplus$

RESUMO: Objetivo: Identificar a capacidade funcional de pacientes atendidos no ambulatório de avaliação perioperatória do Hospital Regional do Gama (APA-HRG), no Distrito Federal, bem como estabelecer relação com as estratificações cardíacas utilizadas. Método: Estudo observacional, descritivo, retrospectivo, com coleta de dados dos registros nos prontuários das consultas pré-operatórias de 292 pacientes triados como alto risco, executadas por equipe de médicos anestesiologistas e enfermeiros, realizadas no APA-HRG no período de junho de 2014 a junho de 2016. Resultados: O perfil da amostra constituiu-se, em sua maioria, por indivíduos do gênero feminino (78,77\%), maiores de 60 anos (48,35\%), não obesos (69,44\%), encaminhados principalmente pela clínica ginecológica (39,79\%), diagnosticados com hipertensão arterial sistêmica (44,17\%) e tabagistas (12,67\%). A capacidade funcional foi classificada como excelente em $63,18 \%$ (>10 equivalentes metabólicos) dos pacientes. Foi constatada associação significativa entre os equivalentes metabólicos e as estratificações da American Society of Anesthesiologists, do Índice de Risco Cardíaco Revisado e da Classificação Funcional da New York Heart Association. Conclusão: A maioria dos prontuários analisados era de pacientes com excelente capacidade funcional, apresentando associação significativa com as estratificações estudadas. Palavras-chave: Idoso. Teste de esforço. Assistência perioperatória. Equipe de assistência ao paciente.

ABSTRACT: Objective: To identify the functional capacity of patients attended at the perioperative evaluation outpatient clinic of the Regional Hospital of Gama (APA-HRG), Federal District, as well as establish relationship with the cardiac stratifications employed. Method: Observational, descriptive, retrospective study with data collection of records from preoperative consultations of 292 patients screened as high risk, performed by a team of medical anesthesiologists and nurses, performed in the APA-HRG in the period from June 2014 to June 2016. Results: The sample profile consisted, for the most part, of female subjects (78.77\%), of over 60 years of age (48.35\%), not obese (69.44\%), referred mainly by the gynecological clinic (39.79\%), diagnosed with systemic arterial hypertension (44.17\%) and smokers (12.67\%). Functional capacity was classified as excellent in $63.18 \%$ (>10 metabolic equivalents) of patients. There was a significant association between the metabolic equivalents and the stratification of the American Society of Anesthesiologists, the Revised Cardiac Risk Index and the Functional Classification of the New York Heart Association. Conclusion: Most of the charts analyzed were of patients with excellent functional capacity, presenting a significant association with the stratifications studied. Key words: Aged. Exercise test. Perioperative care. Patient Care Team.

\footnotetext{
'Enfermeira; doutora em Ciências da Saúde. Coordenadora da Residência de Enfermagem em Centro Cirúrgico em Rede da Escola Superior de Ciências da Saúde da Fundação de Ensino e Pesquisa em Ciências da Saúde (ESCS-FEPECS) Secretaria da Saúde do Distrito Federal (SES/DF) - Brasília (DF), Brasil.

2Enfermeira; especialista em Centro Cirúrgico pela ESCS-FEPECS da SES/DF - Brasília (DF), Brasil.

${ }^{3}$ Médico anestesiologista. Chefe da Unidade de Anestesiologia e Medicina Perioperatória do Hospital Regional do Gama - Brasília (DF), Brasil.

¿Enfermeira; especialista em Centro Cirúrgico pela ESCS-FEPECS da SES/DF - Brasília (DF), Brasil.

${ }^{5}$ Enfermeiro; especialista em Centro Cirúrgico pela ESCS-FEPECS da SES/DF - Brasília (DF), Brasil.

${ }^{6}$ Enfermeira. Residente de Enfermagem em Centro Cirúrgico da ESCS-FEPECS da SES/DF - Brasília (DF), Brasil.

${ }^{7}$ Major do Quadro Oficial de Bombeiros Militar. Médico anestesiologista do Corpo de Bombeiros Militar do Distrito Federal (CBMDF) - Brasília (DF), Brasil.

*Autora correspondente: jacquelinecchbdf@gmail.com

Recebido: 08/06/2018 - Aprovado: 09/03/2019
}

DOl: 10.5327/Z1414-4425201900030003 
RESUMEN: Objetivo: Identificar la capacidad funcional de pacientes atendidos en el ambulatorio de evaluación perioperatoria del Hospital Regional do Gama (APA-HRG), en el Distrito Federal, así como establecer relación con las estratificaciones cardíacas utilizadas. Método: Estudio observacional, descriptivo, retrospectivo, con colecta de datos de los registros en los historiales médicos de las consultas preoperatorias de 292 pacientes seleccionados como alto riesgo, ejecutadas por equipo de médicos anestesistas y enfermeros, realizadas en el APA-HRG en el período de junio de 2014 a junio de 2016. Resultados: El perfil de la muestra se constituye, en su mayoría, por individuos del género femenino (78,77\%), mayores de 60 años (48,35\%), no obesos $(69,44 \%)$, encaminados principalmente por la clínica ginecológica (39,79\%), diagnosticados con hipertensión arterial sistémica (44,17\%) y tabaquistas (12,67\%). La capacidad funcional fue clasificada como excelente en un $63,18 \%$ (>10 equivalentes metabólicos) de los pacientes. Fue constatada asociación significativa entre los equivalentes metabólicos y las estratificaciones de la American Society of Anesthesiologists, del Índice de Riesgo Cardíaco Revisado y de la Clasificación Funcional de la New York Heart Association. Conclusión: La mayoría de los historiales médicos analizados era de pacientes con excelente capacidad funcional, presentando asociación significativa con las estratificaciones estudiadas.

Palabras-clave: Anciano. Prueba de esfuerzo. Atención perioperativa. Grupo de atención al paciente.

\section{INTRODUÇÃo}

Segundo o Instituto Brasileiro de Geografia e Estatística (IBGE) ${ }^{1}$, o segmento da população brasileira que mais cresce é o de idosos, com projeção de 44,44\% para o ano de 2060. Acredita-se que em 2025 o país esteja em sexto lugar na classificação mundial em número de idosos ${ }^{2}$. Em consequência, o volume de cirurgias não cardíacas em pacientes idosos, associados a comorbidades, tem aumentado. Esse fato culmina em maior risco de morbidade e mortalidade no pós-operatório ${ }^{3}$.

Entre os principais fatores de risco relacionadas à morte em cirurgias não cardíacas encontram-se as doenças cardiovasculares, principalmente a doença arterial coronariana $(\mathrm{DAC})^{4-6}$. Tal evidência aponta para a necessidade de absorver essa crescente demanda por avaliações perioperatórias multiprofissionais, a fim de identificar fatores que aumentam o risco cirúrgico e planejar estratégias que o reduza, visando a um resultado cirúrgico satisfatório ${ }^{7,8}$. Para tanto, determina-se a capacidade funcional de cada paciente, que é uma preditora confiável do risco cardíaco e que ajuda a determinar a necessidade de testes adicionais e terapia farmacológica específica antes e durante a cirurgia planejada ${ }^{9}$.

Uma boa capacidade funcional geralmente é definida com base nos níveis de equivalência metabólica (MET). Uma unidade MET é definida como $3,5 \mathrm{~mL} / \mathrm{kg} / \mathrm{min}$ e representa o consumo basal de oxigênio de um homem médio de $70 \mathrm{~kg}$ em repouso ${ }^{10}$. Para fins de referência, as atividades da vida diária, como comer, vestir, usar o banheiro e tomar banho, geralmente requerem 1 a 2 MET, enquanto esportes tão intensos como natação, tênis e esqui exigem mais de $10 \mathrm{MET}^{11}$.

Para avaliar a capacidade funcional do paciente no período perioperatório, o teste ergométrico se mostra um exame de pouco custo financeiro, fácil execução e alta reprodutibilidade, compatível com a realidade de várias regiões e municípios do Brasil. É notável que o gradiente de gravidade no teste tem enorme correspondência com a evolução perioperatória. O início da resposta isquêmica, quando em baixa carga, apresenta relação quanto ao aumento de eventos cardíacos perioperatórios ${ }^{5,12}$. A realização desse teste não está indicada para o grupo de pacientes de baixíssimo risco, pois não acrescenta benefício, nem para pacientes de alto risco devido à necessidade de estratificação invasiva ${ }^{5}$.

Outra maneira utilizada para determinar a tolerância ao exercício de um paciente é a anamnese. Os pacientes são convidados a descrever a natureza e a frequência de suas atividades físicas. Essa é outra informação, alternativa ao teste formal, para avaliar a capacidade do paciente de se submeter a uma cirurgia com segurança ${ }^{13}$.

Perguntas simples permitem uma estimativa razoável da capacidade funcional do paciente. Por exemplo, pode-se perguntar se o paciente consegue andar quatro blocos sem parar devido a sintomas limitantes e escalar dois andares de escadas sem parar em razão de sintomas limitantes. Respostas afirmativas confirmam capacidade funcional adequada, pois tais atividades suportam a tolerância do paciente ao esforço em um nível de 4 a 5 MET, o equivalente ao estresse cirúrgico ${ }^{10}$.

Em contrapartida, respostas negativas a ambas as questões são geralmente associadas a pacientes mais velhos, propensos a ter diabetes, doença pulmonar obstrutiva, insuficiência cardíaca congestiva, hipertensão e maiores valores de classificação de estado físico da American Society of Anesthesiologists (ASA). Além disso, preveem maior probabilidade de má evolução pós-operatória ${ }^{13}$.

Considerando as características específicas do paciente cirúrgico e a importância dos esforços para a obtenção da melhoria da qualidade da assistência prestada ao paciente, o ambulatório de avaliação perioperatória de um hospital geral é um serviço voltado para a avaliação perioperatória. Os pacientes 
são atendidos por uma equipe multiprofissional (anestesiologistas e equipe de enfermagem) que, fundamentados em práticas baseadas em evidências, provêm cuidados perioperatórios individualizados ao paciente, em busca de melhores resultados.

$\mathrm{Na}$ consulta de avaliação perioperatória, realizada no ambulatório de avaliação perioperatória do Hospital Regional do Gama (APA-HRG), o enfermeiro, juntamente com o médico anestesiologista, aplica as estratificações de risco de síndrome da apneia obstrutiva do sono (score STOP-BANG), capacidade funcional (MET) e risco de trombose venosa (safety zone). O anestesiologista utiliza também a classificação do estado físico da ASA e os riscos cardíacos pela classificação funcional da New York Heart Association (NYHA), Índice de Risco Cardíaco Revisado de Lee (IRCR) e risco cardíaco para procedimentos não cardíacos ${ }^{14,15}$.

\section{OBJETIVO}

Identificar a capacidade funcional de pacientes atendidos no APA-HRG, no Distrito Federal, bem como estabelecer relação com as estratificações cardíacas utilizadas.

\section{MÉTODO}

Trata-se de um estudo observacional, descritivo, retrospectivo, com coleta de dados das consultas realizadas no período de junho de 2014 a junho de 2016 no APA-HRG, um hospital regional situado em Brasília, Distrito Federal.

A população consistiu nos prontuários de pacientes candidatos à cirurgia eletiva encaminhados pelas especialidades cirúrgicas ao APA-HRG. A amostra contou com 292 prontuários de pacientes triados como alto risco. Foram considerados pacientes de alto risco, segundo o protocolo do serviço, os que possuíam mais de 65 anos, ou menos de 65 anos com antecedentes mórbidos (alergias, doenças sistêmicas, uso de medicações de forma contínua) e/ou limitação física e/ou limitação cognitiva e/ ou história prévia de intercorrência em procedimento cirúrgico-anestésico.

Foram incluídos no estudo os prontuários (físicos e eletrônicos) de pacientes maiores de 18 anos, com doenças cirúrgicas de tratamento eletivo, encaminhados pelas clínicas de cirurgia geral e subespecialidades, ginecologia e ortopedia, avaliados pelo APA-HRG. Os critérios de exclusão foram os prontuários dos pacientes cuja cirurgia não foi realizada até o mês de junho de 2017.

Do total da amostra, foram incluídos 50 prontuários de pacientes atendidos no segundo semestre de 2014, 125 em 2015 e 117 no primeiro semestre de 2016.

Para a coleta de dados, utilizou-se um formulário que reunia as principais informações contidas no questionário padronizado para consultas pré-operatórias do serviço, registradas no prontuário físico ou eletrônico dos pacientes. Além das variáveis sociodemográficas e antropométricas (sexo, idade, índice de massa corporal, comorbidades e hábitos tabágicos), foram coletadas a classificação do estado físico pela ASA e a classificação funcional pelo MET (Quadro 1), assim como as avaliações de risco cardíaco: Lee, NYHA e risco cardíaco para procedimentos não cardíacos.

$\mathrm{O}$ instrumento utilizado para avaliar a capacidade funcional foi retirado dos atuais Guidelines do American College of Cardiology/American Heart Association (ACC/AHA), adaptado do Duke Activity Status Index ${ }^{16}$.

A análise dos dados foi realizada por meio de estatística descritiva dos dados socioeconômicos e demográficos, além da comparação das estratificações clínicas e cardíacas com a capacidade funcional (análise inferencial).

As variáveis categóricas foram descritas por meio de frequências absolutas (n) e relativas (\%) e as associações foram testadas mediante o teste do $c^{2}$ com nível de significância de $5 \%(\mathrm{p}<0,05)$. As variáveis foram analisadas por meio do emprego do software estatístico R ( $\mathrm{R}$ Development Core Team 2008 versão 3.3 para Windows $\left.{ }^{\circledR}\right)$.

Em relação à análise crítica dos riscos, pelo fato de a pesquisa utilizar apenas prontuários físicos ou eletrônicos, os riscos referentes ao trabalho foram mínimos. $\mathrm{O}$ anonimato dos pacientes foi assegurado, pois o estudo teve enfoque nos dados em geral e não nos pacientes individualmente.

O projeto foi aprovado pelo Comitê de Ética em Pesquisa da Fundação de Ensino e Pesquisa em Ciências da Saúde (FEPECS),

Quadro 1. Classificação da capacidade funcional em níveis de equivalência metabólica (MET) utilizada pelo ambulatório de avaliação perioperatória do Hospital Regional do Gama.

\begin{tabular}{|l|c|}
\hline $\begin{array}{l}\text { Equivalente } \\
\text { metabólico (MET) }\end{array}$ & Tipo de atividade \\
\hline Excelente (>7 MET) & $\begin{array}{c}\text { Pratica futebol, natação, tênis, } \\
\text { corrida de curtas distâncias }\end{array}$ \\
\hline Moderada (4 a 7 MET) & $\begin{array}{c}\text { Caminhada com } \\
\text { velocidade de } 6,4 \mathrm{~km} / \mathrm{h}\end{array}$ \\
\hline Ruim (<4 MET) & $\begin{array}{c}\text { Pouca atividade, caminhadas curtas } \\
\text { (duas quadras), com velocidade, } \\
\text { no máximo, de } 4,8 \mathrm{~km} / \mathrm{h}\end{array}$ \\
\hline MET: 0 consumo de oxigênio $\left(\mathrm{VO}_{2}\right)$ & de um homem de 40 anos, com $70 \mathrm{Kg}$ em repouso é de \\
$3,5 \mathrm{~mL} / \mathrm{kg}$, ou o correspondente 1 MET. Fonte: Fleisher et al.?.
\end{tabular}


sob número CAAE 60740916.8.0000.5553, sendo realizado em conformidade com as exigências da Resolução $n^{\circ}$ 466, de 12 de dezembro de 2012, do Conselho Nacional de Saúde (CNS).

\section{RESULTADOS}

O perfil da amostra estudada foi composto, em sua maioria, de indivíduos do gênero feminino $(78,77 \%)$, maiores de 60 anos (48,35\%), não obesos (69,44\%), encaminhados principalmente pela clínica ginecológica $(39,79 \%)$, diagnosticados

Tabela 1. Variáveis demográficas e antropométricas da população pesquisada atendida pelo ambulatório de avaliação perioperatória do Hospital Regional do Gama. Distrito Federal, 2016.

\begin{tabular}{|c|c|c|}
\hline Variáveis & $\mathbf{n}$ & $\%$ \\
\hline \multicolumn{3}{|l|}{ Sexo } \\
\hline Feminino & 230 & 78,77 \\
\hline Masculino & 62 & 21,23 \\
\hline \multicolumn{3}{|l|}{ Idade (anos) } \\
\hline$<20$ & 01 & 0,41 \\
\hline $20 \mathrm{~A} 40$ & 41 & 16,94 \\
\hline $41 \mathrm{~A} 60$ & 83 & 34,3 \\
\hline$>60$ & 117 & 48,35 \\
\hline \multicolumn{3}{|l|}{ Imc $\left(\mathrm{Kg} / \mathrm{m}^{2}\right)$} \\
\hline$<30$ & 200 & 69,44 \\
\hline$>30$ & 88 & 30,56 \\
\hline \multicolumn{3}{|l|}{ Especialidade } \\
\hline Geral & 68 & 23,53 \\
\hline Ginecologia & 115 & 39,79 \\
\hline Mastologia & 05 & 1,73 \\
\hline Trauma e ortopedia & 85 & 29,41 \\
\hline Vascular & 06 & 2,08 \\
\hline Outras & 10 & 3,46 \\
\hline \multicolumn{3}{|l|}{ Doenças associadas } \\
\hline Has & 125 & 44,17 \\
\hline $\mathrm{Dm}$ & 12 & 4,24 \\
\hline Has+dm & 35 & 12,37 \\
\hline Não & 111 & 39,22 \\
\hline \multicolumn{3}{|l|}{ Tabagismo } \\
\hline Sim & 37 & 12,67 \\
\hline Não & 200 & 68,49 \\
\hline Ex-tabagista & 55 & 18,84 \\
\hline
\end{tabular}

IMC: Índice de massa corporal; HAS: hipertensão arterial sistêmica; DM: diabetes mellitus. com hipertensão arterial sistêmica (HAS) $(44,17 \%)$ e tabagistas $(12,67 \%)$, conforme demonstrado na Tabela 1.

Em relação à capacidade funcional, 277 prontuários de pacientes foram avaliados e desses, 11 (3,97\%) obtiveram capacidade funcional ruim (1 a 4 MET), em contraste com 175 $(63,18 \%)$ que foram classificados como excelente (>10 MET), conforme dados da Tabela 2.

Quando se estabeleceu relação entre o MET, o sistema de classificação do estado físico do paciente (ASA) e as estratificações de risco cardíaco (Lee e NYHA), verificou-se, pelos valores p, associação significativa em todos os casos. Isto é, a depender da classificação do MET, a classificação das estratificações também é alterada (Tabela 3).

$\mathrm{Na}$ Tabela 4, ao analisar a relação entre o MET e o risco cardíaco pelo procedimento não cardíaco, $44,76 \%$ dos pacientes obtiveram MET excelente e iriam ser submetidos a procedimentos não cardíacos considerados de risco intermediário,

Tabela 2. Avaliação do equivalente metabólico na população atendida pelo ambulatório de avaliação perioperatória do Hospital Regional do Gama. Distrito Federal, 2016.

\begin{tabular}{|l|c|c|}
\hline MET & $\mathbf{n}$ & $\%$ \\
\hline Excelente & 175 & 63,18 \\
\hline Moderado & 91 & 32,85 \\
\hline Ruim & 11 & 3,97 \\
\hline
\end{tabular}

MET: níveis de equivalência metabólica.

Tabela 3. Teste do $\chi^{2}$ entre o equivalente metabólico (MET) e as estratificações realizadas pelo ambulatório de avaliação perioperatória do Hospital Regional do Gama. Distrito Federal, 2016.

\begin{tabular}{|l|c|c|c|} 
& $\chi^{2^{*}}$ & Graus de liberdade & $\mathbf{p}$ \\
\hline ASA & 37,638 & 4 & 0,000 \\
\hline LEE & 31,284 & 6 & 0,000 \\
\hline NYHA & 22,661 & 4 & 0,000 \\
\hline
\end{tabular}

ASA: American Association of Anaesthesia; LEE: índice de risco cardíaco revisado; NYHA: New York Heart Association; * programa SPSS versão 25.0.0.0.

Tabela 4. Relação entre o equivalente metabólico e o risco cardíaco pelo procedimento não cardíaco realizado pelo ambulatório de avaliação perioperatória do Hospital Regional do Gama. Distrito Federal, 2016.

\begin{tabular}{|l|c|c|c|}
\multirow{2}{*}{ MET } & \multicolumn{3}{|c|}{ Risco cardlíaco pelo procedimento não cardíaco (\%) } \\
\cline { 2 - 4 } & Baixo & Intermediário & Alto \\
\hline Excelente & 15,89 & 44,76 & 2,52 \\
\hline Moderado & 5,41 & 26,35 & 1,09 \\
\hline Ruim & 0,72 & 2,89 & 0,36 \\
\hline
\end{tabular}

MET: equivalente metabólico. 
assim como os $26,35 \%$ que obtiveram MET moderado. Esse grupo de pacientes soma a maioria da amostra e eles foram liberados para cirurgia com segurança, sem precisar de outros testes, seguindo as diretrizes do ACC/AHA.

\section{DISCUSSÃO}

No presente estudo, a maioria da amostra pertencia ao gênero feminino $(78,77 \%)$ e tinha mais de 60 anos $(48,35 \%)$. O processo de envelhecimento populacional no Brasil tem ocorrido em todo o país, com maior sobrevivência da população feminina, que alcançou uma expectativa de vida ao nascer de 72,6 anos em 2000, quase 8 anos a mais que a dos homens. Por esse motivo, também, a ginecologia foi a especialidade mais atendida $(39,7 \%)$ neste estudo ${ }^{17}$.

O estudo também mostrou a maior incidência de pacientes com diagnóstico de HAS $(44,17 \%)$ em relação às patologias associadas. De forma semelhante ocorreu em outra pesquisa, na qual $71,3 \%$ dos pacientes avaliados no pré-operatório apresentavam doenças associadas ${ }^{18}$. Concomitantemente ao processo de envelhecimento populacional, o aumento da prevalência das doenças crônicas não transmissíveis demanda a necessidade de tratamentos contínuos e aumento da ocorrência de graus variáveis de disfunções e dependências, o que traz implicações diretas ao atendimento do paciente cirúrgico ${ }^{18}$.

Um número expressivo de pacientes obesos $(30,56 \%$ com índice de massa corporal $>30 \mathrm{~kg} / \mathrm{m}^{2}$ ) foi encontrado neste estudo, o que exacerba com quadro nacional, no qual a prevalência é $19,6 \%$ entre mulheres e $18,1 \%$ entre homens no Brasil $^{19}$. Sabe-se que esse dado é relevante para o delineamento do risco cirúrgico, visto que a obesidade é fator condicionante para doença cardiovascular ${ }^{20}$.

Em relação ao tabagismo, a maioria da amostra $(68,49 \%)$ não era tabagista, um dado esperado, de acordo com a realidade brasileira. Segundo dados de 2016 da Vigilância de Fatores de Risco e Proteção para Doenças Crônicas por Inquérito Telefônico (VIGITEL), o percentual de fumantes maiores de 18 anos no Brasil é de 10,2\%, sendo 12,7\% entre homens e $8 \%$ entre mulheres ${ }^{19}$.

A capacidade funcional foi classificada como excelente em $63,18 \%$ (>10 MET) dos pacientes. Esse resultado era esperado, pois segundo a coleta do VIGITEL 2016 no Distrito Federal, a prática de atividade física no tempo livre aumentou aproximadamente $7,3 \%$ entre os anos de 2009 e 2016, apesar de a prevalência de atividade física ainda reduzir conforme a idade ${ }^{19}$.

Segundo dados do VIGITEL, em 2016 a frequência de indivíduos fisicamente inativos foi de $10,3 \%$ no Distrito
Federal, a mais baixa dentre todas as capitais brasileiras ${ }^{19}$. Além disso, do total de moradores da região administrativa do Gama analisados pela Pesquisa Distrital por Amostra de Domicílios (PDAD/DF), 64,50\% contam com Ponto de Encontro Comunitário (PEC), 48,85\% com ciclovia e 29,64\% têm ruas arborizadas próximas de suas residências ${ }^{21}$, o que possivelmente colabora para o estilo de vida ativo da população.

Somente $3,9 \%$ da amostra foram classificados com a capacidade funcional ruim ( $<4 \mathrm{MET})$. Esses pacientes estão sob risco aumentado para complicações cardíacas operatórias. Porém, sabe-se que a má condição física é um fator de risco modificável e melhorias na aptidão ao longo do tempo foram demonstradas para melhorar o prognóstico. Um estudo concluiu que cada incremento de 1 MET na capacidade de exercício é associada a reduções de mortalidade de aproximadamente $12 \%$, sendo, independentemente da forma de mensuração, um poderoso preditor de risco cardiovascular ${ }^{22}$.

Grande parte dos pacientes com doença cardiovascular estabelecida refere diminuição da capacidade funcional ${ }^{23}$. E discriminar objetivamente o risco cirúrgico para cada condição específica da doença arterial coronariana (DAC) é fundamental para a prevenção e para uma menor morbidade de eventos perioperatórios ${ }^{5}$. Este trabalho demonstrou, então, que existe associação significativa entre o MET e algumas das estratificações cardíacas preconizadas pela Sociedade Brasileira de Cardiologia: ASA, Lee e NYHA.

Um estudo realizado com 1.049 pacientes verificou que a sobrevivência pós-operatória de 30 dias e em longo prazo foi significativamente melhor, independentemente do tipo de cirurgia, em ASA P3 com independência funcional do que naqueles ASA P3 com capacidade funcional limitada ${ }^{24}$.

Nos pacientes portadores de insuficiência cardíaca, evidenciados na classificação NYHA, a perda da capacidade funcional está relacionada à menor fração de ejeção e ao menor débito cardíaco. Já nos portadores de cardiopatia isquêmica, evidenciados no IRCR, o exercício também é limitado pelo eventual desencadeamento de isquemia miocárdica ${ }^{23}$.

Segundo o protocolo do ACC/AHA, após determinar o estado físico e o risco cardíaco do paciente (passo 1) e estimar sua capacidade funcional (passo 2), deve-se considerar o risco cardíaco relacionado aos diversos tipos de procedimentos cirúrgicos (passo 3$)^{25}$.

Neste estudo, a maioria (74\%) dos procedimentos cirúrgicos foi classificada como risco intermediário. O risco intrínseco do procedimento cirúrgico corresponde à probabilidade de ocorrência de eventos cardiovasculares perioperatórios ${ }^{5}$. Os procedimentos cirúrgicos extensivos, em particular aqueles 
no abdômen ou no tórax e aqueles associados com grandes mudanças no volume e/ ou perda de sangue têm maiores riscos ${ }^{25}$. Foram classificados como risco baixo $22,02 \%$. Esses pequenos procedimentos ambulatoriais estão associados a uma baixíssima taxa de morbidade e mortalidade ${ }^{26}$.

Quando o paciente apresenta preditores de risco cardíaco menores, com capacidade funcional moderada ou excelente, e se o procedimento for de risco intermediário ou baixo, a cirurgia pode ser liberada ${ }^{8}$. Sendo assim, $44,76 \%$ dos pacientes que obtiveram MET excelente e iriam ser submetidos a procedimentos não cardíacos considerados de risco intermediário, assim como os $26,35 \%$ que obtiveram MET moderado, foram liberados para cirurgia com segurança, sem precisar de outros testes, seguindo as diretrizes do ACC / $\mathrm{AHA}^{25}$.

A incorporação da avaliação da capacidade funcional na consulta de enfermagem perioperatória traz ao enfermeiro e aos demais profissionais da equipe multiprofissional uma investigação de risco mais completa, já que o MET é um importante preditor de mortalidade.

Neste estudo avaliamos o MET por meio do questionário, por ser uma ferramenta mais prática e acessível. Assim sendo, outros trabalhos, incluindo o teste ergométrico para verificação do MET, devem ser incentivados.

\section{CONCLUSÃO}

Com este estudo foi possível observar que o APA-HRG segue, em suas práticas, as atuais diretrizes de avaliação perioperatória preconizadas pelo ACC/AHA.

O estudo evidenciou predominância de pacientes do gênero feminino, maiores de 60 anos, não obesos, tabagistas, diagnosticados com HAS, encaminhados, em maior incidência, pela clínica ginecológica.

A população deste estudo, em sua maioria, teve a capacidade funcional classificada como excelente e estaria sob menor risco cardiovascular. Também foi verificada associação significativa entre a avaliação da capacidade funcional (MET) e as estratificações ASA, Lee e NYHA. Sendo assim o MET, compondo uma avaliação perioperatória baseada nas melhores evidências científicas, fornece aos enfermeiros e anestesiologistas informações importantes para o prosseguimento de cirurgias não cardíacas com segurança.

Verifica-se, ainda, a necessidade de outros estudos sobre a avaliação da capacidade funcional em paciente pré-cirúrgicos, tendo em vista que são escassos os trabalhos sobre esse tema na literatura brasileira.

\section{REFERÊNCIAS}

1. Instituto Brasileiro de Geografia e Estatística. Coordenação de População do IBGE. Mudança Demográfica no Brasil no Início do Século XXI: subsídios para as projeções da população do Brasil e das Unidades da Federação [Internet]. Brasil: Instituto Brasileiro de Geografia e Estatística; 2017 [acessado em 09 jun. 2017. Disponível em: https://agenciadenoticias.ibge.gov.br/agencia-noticias/2013agencia-de-noticias/releases/9831-ibge-lanca-estudo-metodologicosobre-mudanca-demografica-e-projecoes-de-populacao.html

2. Machado WD, Gomes DF, Freitas CASL, Brito MCC, Moreira ACA. Elderly with not transmitted chronic diseases: a group association study. Reon Facema [Internet]. 2017 [acessado em 09 jun. 2017;3(2):444-51. Disponível em: http://www.facema.edu.br/ojs/index.php/ReOnFacema/ article/view/194/106

3. Reis PV, Souza GS, Lopes AM, Costa AV, Santos A, Abelha FJ. Severity of disease scoring systems and mortality after non-cardiac surgery. Braz J Anesthesiol. 2018;68(3):244-53. https://doi.org/10.1016/j.bjan.2017.12.001

4. Ramos GC. Aspectos relevantes da doença arterial coronariana em candidatos à cirurgia não cardíaca. Rev Bras Anestesiol. 2010;60(6):6625. http://dx.doi.org/10.1590/S0034-70942010000600013

5. Gualandro DM, Yu PC, Caramelli B, Marques AC, Calderaro D, Fornari LS, et al. 3a Diretriz de Avaliação Cardiovascular Perioperatória da Sociedade Brasileira de Cardiologia. Arq Bras Cardiol. 2017;109(3 Supl. 1):1-104.
6. Cesar LA, Ferreira JF, Armaganijan D, Gowdak LH, Mansur AP, Bodanese LC, et al. Diretriz de Doença Coronária Estável. Arq Bras Cardiol. 2014;103(2 Supl. 2):1-59.

7. Pontes SRS, Salazar RM, Torres OJM, TCBC-MA. Avaliação perioperatória de pacientes em unidade de terapia intensiva. Rev Col Bras Cir. 2013;40(2). http://dx.doi.org/10.1590/ S0100-69912013000200002

8. Fernandes EO, Guerra EE, Pitrez FAB, Fernandes FM, Rosito GBA, Gonzáles HE, et al. Avaliação pré-operatória e cuidados em cirurgia eletiva: recomendações baseadas em evidências. Rev AMRIGS. 2010;54(2):240-58.

9. Fleisher LA, Beckman JA, Brown KA, Calkins H, Chaikof E, Fleischmann KE, et al. ACC/AHA 2007 Guidelines on perioperative cardiovascular evaluation and care for noncardiac surgery: a report of the American College of Cardiology/American Heart Association Task Force on Practice Guidelines (Writing Committee to Revise the 2002 Guidelines on Perioperative Cardiovascular Evaluation for Noncardiac Surgery): developed in collaboration with the American Society of Echocardiography, American Society of Nuclear Cardiology, Heart Rhythm Society, Society of Cardiovascular Anesthesiologists, Society for Cardiovascular Angiography and Interventions, Society for Vascular Medicine and Biology, and Society for Vascular Surgery. Circulation. 2007;116(17):e418-99. https://doi.org/10.1161/CIRCULATIONAHA.107.185699 
10.Sabiston DC. Tratado de cirurgia. 19a ed. Rio de Janeiro: Elsevier; 2014.

11. Prefeitura do Rio de Janeiro. Secretaria Municipal de Saúde. Avaliação pré-operatória. Coleção Guia de Referência Rápida SMS - RJ/SUBPAV/ SAP. Rio de Janeiro: Prefeitura do Rio de Janeiro; 2016.

12. Feitosa ACR, Marques AC, Caramelli B, Ayub B, Polanczyk CA, Jardim C, et al. II Diretriz de Avaliação Perioperatória da Sociedade Brasileira de Cardiologia. Arq Bras Cardiol. 2011;96(3 Supl. 1):1-68.

13. Reilly DF, McNeely MJ, Doerner D, Greenberg DL, Staiger TO, Geist $\mathrm{MJ}$, et al. Self-reported exercise tolerance and the risk of serious perioperative complications. Arch Intern Med. 1999;159(18):2185-92.

14. Loureiro BMC, Feitosa-Filho GS. Escores de risco perioperatório para cirurgias não-cardíacas: descrições e comparações. Rev Soc Bras Clin Med. 2014;12(4):314-20.

15. Santos IS, Bittencourt MS. Heart failure. Rev Med [Internet]. 2008 [acessado em 10 nov. 2017];87(4):224-31. Disponível em: https:// www.revistas.usp.br/revistadc/article/download/59083/62069

16. Hlatky MA, Boineau RE, Higginbotham MB, Lee KL, Mark DB, Califf $\mathrm{RM}$, et al. A brief self-administered questionnaire to determine functional capacity (the Duke Activity Status Index). Am J Cardiol. 1989;64(10):651-4.

17. Leão EM, Marinho LFB. Saúde das mulheres no Brasil: subsídios para as políticas públicas de saúde. Rev Promoção Saúde. 2002;6:31-6.

18. Santos ML, Novaes CO, Iglesias AC. Epidemiological profile of patients seen in the pre-anesthetic assessment clinic of a university hospital. Braz J Anesthesiol. 2017;67(5):457-67. https://doi.org/10.1016/j. bjan.2016.06.002
19. Brasil. Ministério da Saúde. Secretaria de Vigilância em Saúde. Departamento de Vigilância de Doenças e Agravos não Transmissíveis e Promoção da Saúde. Vigitel Brasil 2016: vigilância de fatores de risco e proteção para doenças crônicas por inquérito telefônico: estimativas sobre frequência e distribuição sociodemográfica de fatores de risco e proteção para doenças crônicas nas capitais dos 26 estados brasileiros e no Distrito Federal em 2016. Brasília: Ministério da Saúde; 2017.

20. Vargas BD, Sangiovo A, PereiraF, Vincensi C, Lissarassa YPS, Zimmermann CA, et al. Obesidade, diabetes, hipertensão associados ao desenvolvimento de dano renal e redução na qualidade de vida. Rev Saúde Integrada. 2016;9(18).

21. Distrito Federal. Governo do Distrito Federal. Secretaria de Estado de Planejamento, Orçamento e Gestão. Companhia de Planejamento do Distrito Federal. Pesquisa Distrital por Amostra de Domicilios (GAMA). PDAD 2015. Brasilia: Companhia de Planejamento do Distrito Federal; 2015.

22. Myers J, Prakash M, Froelicher VD, Do D, PartingtonS, Atwood J. Exercise capacity and mortality among men referred for exercise testing. N Engl J Med. 2002;346(11):793-80. https://doi.org/10.1056/NEJMoa011858

23. Moraes RS, Nóbrega ACL, Castro RRT, Negrão CE, Stein R, Serra SM, et al. Diretriz de Reabilitação Cardíaca. Arq Bras Cardiol. 2005;84(5):43140. http://dx.doi.org/10.1590/S0066-782X2005000500015

24. Visnjevac O, Lee J, Pourafkari L, Dosluoglu HH, Nader ND, KritchevskyS. Functional capacity as a significant independent predictor of postoperative mortality for octogenarian ASA-III patients. J Gerontol A Biol Sci Med Sci. 2014;69(10):1229-35. https://doi.org/10.1093/gerona/glu062

25. Mukherjee D, Eagle KA. Perioperative cardiac assessment for noncardiac surgery. Circulation. 2003;107(22):2771-4. https://doi. org/10.1161/01.CIR.0000072248.24921.D6

26. Novaes MV. Avaliação e preparo pré-operatório: classificação do estado físico. Medicina Perioperatória. Rio de Janeiro: SAERJ; 2006. 